\title{
Satisfacción en el noviazgo en adultos jóvenes según su orientación sexual en Mérida, Yucatán
}

Dating Satisfaction in young adults according to their sexual orientation in Merida, Yucatan

\begin{abstract}
Génesis Cahuich Lemus
Jessica Chacón Alvarado

Mayra Juárez Mendoza

Leidy Kantún López

Luz Vázquez González

\section{Resumen}

Las relaciones heterosexuales han sido más aceptadas que las relaciones de diferente orientación sexual, sin embargo, no se puede ignorar la existencia de estas últimas en una relación de noviazgo. La presente investigación busca establecer si existe relación entre la satisfacción en el noviazgo, dependiendo de la orientación sexual y el género, en un grupo de adultos jóvenes. Se utilizó la Escala Multidimensional de Satisfacción en el Noviazgo de Bargas y García (2011), compuesta por 11 dimensiones. La muestra estuvo constituida por 90 participantes de 18 a 30 años de los cuales fueron 44 hombres y 46 mujeres. En cuanto a la orientación sexual, 47 dijeron ser heterosexuales, y 43 con una orientación sexual diferente. Los resultados según la orientación sexual y la satisfacción en el noviazgo sugieren que las personas con orientación heterosexual son los más satisfechos en las dimensiones de interacción familiar que los de otra orientación sexual. Respecto a la satisfacción en hombres y mujeres independientemente de su orientación sexual, los resultados sugieren que los hombres están más satisfechos que las mujeres en lo que se refiere a pasar tiempo juntos y compartir actividades, y las mujeres están más satisfechas respecto de la interacción con los amigos de su pareja, en la toma de decisiones, acuerdos y la comunicación con la pareja. Se discute sobre la influencia de las relaciones familiares en el noviazgo debido a que si no existe la aceptación el distanciamiento familiar puede afectar la satisfacción en la pareja.
\end{abstract}

\section{Abstract}

Heterosexual relationships have been more accepted than homosexual orientation, however, it cannot be ignored the existence of the latter in a dating relationship. This research seeks to establish if there is a relationship between dating satisfaction, depending on sexual orientation and gender, in a group of young adults. The Multidimensional scale of dating satisfaction of Bargas and Garcia (2011) composed of 11 dimensions was used. The sample consisted of 90 participants from 18 to 30 years of which 44 were men and 46 women. In terms of sexual orientation, 47 claimed to be heterosexual, and 43 with a different sexual orientation. Results showed that, according to sexual orientation and dating satisfaction, people with heterosexual orientation are the most satisfied in the familiar interaction dimensions than another sexual orientation. With respect to satisfaction of men and women regardless of their sexual orientation, the results suggest that men are happier than women regarding to the time spent together and shared activities, and women are more satisfied concerning to the interaction with their partner's friends, in making decisions, agreements, and communication with the couple. It is discussed the influence of family on dating relationships since the absence of acceptance could cause family distancing and it may affect the couple's satisfaction.

Palabras Clave: Satisfacción, noviazgo, orientación sexual, heterosexualidad, homosexualidad. Keywords: Satisfaction, dating, sexual orientation, heterosexuality, homosexuality. 
Nicolau, Martell y Méndez (2004), consideran que entre la amplia diversidad de conductas presentes a lo largo de la existencia humana, la elección de pareja ocupa un lugar primordial en la vida de muchas personas; elegir una pareja es una de las decisiones más importantes en la vida adulta. Es por eso que el noviazgo constituye una de las relaciones más importantes para la vida emocional de las personas jóvenes, ya que es una de las etapas para el establecimiento de una pareja formal.

El noviazgo es una etapa en la relación de pareja que pretende construir un vínculo amoroso que permita el acercamiento y conocimiento profundo de las partes para definir si es posible establecer una relación de mayor trascendencia como el matrimonio o la unión consensual (Villagómez, 2010). Esta etapa es una de las más importantes en la vida de las personas, dado que es aquella en la cual se crea y se consolida una relación de pareja estable. Además, es durante su transcurso cuando miembros de la pareja van construyendo un aprendizaje acerca de cómo relacionarse, conocen sus diferencias y similitudes, para encarar situaciones difíciles, acoplarse como pareja y establecer pautas de comportamiento para el desarrollo tanto personal como de pareja (López-Parra, Rivera-Aragón, García-Méndez \& Vargas-Núñez, 2010).

Cuando se habla de noviazgo, es importante considerar no sólo los noviazgos heterosexuales, sino también las relaciones que llevan las parejas con otras orientaciones sexuales, de tal forma que se logre una igualdad para todas las personas al abordar este tema. Sin embargo, la sexualidad heterosexual es considerada la natural, buena, necesaria, saludable y moralmente positiva, mientras que el resto de las sexualidades son consideradas contranaturales, malas, negativas, enfermas, moralmente censurables o pecaminosas. Esta heteronormatividad intrínseca del sistema de parentesco es lo que ha impedido, hasta hace poco, que las personas con otras orientaciones sexuales pensaran sus relaciones en términos de familia y matrimonio (Pichardo, 2009).

Un acto fuera de la heterosexualidad no define a la persona como de orientación sexual diferente. Ésta se define por la capacidad de tener una relación sentimental, de enamorarse de alguien del mismo sexo, y poco importa si la relación se consuma o no. Es el hecho de enamorarse lo que define a las personas como bisexuales u homosexuales (Diaz-Loving, 1999). Actualmente, no se considera que tener una orientación sexual distinta a la heterosexualidad sea un pecado, un delito punible por la ley, ni una enfermedad que deba "curarse" como se consideró tiempo atrás. Es una opción de vida y es tan válida como la heterosexualidad, ni mejor ni peor que ella. Además, la orientación sexual se presenta en un continuo, no es algo rígido e invariable y muestra importantes variaciones (Ardila, 2008). Los derechos de los homosexuales deben integrarse dentro de la lucha por los derechos humanos, al igual que los derechos de la mujer, de las diversas etnias, de los grupos minoritarios, etcétera.

Por ello, en cuanto al estudio de la homosexualidad, para Ardila (2008) ésta tiene mucho que ver con aspectos de la diversidad humana y no con la psicopatología. De esta manera, las investigaciones sobre orientaciones sexuales como la homosexualidad o la bisexualidad ayudan a entender al ser humano en su conjunto y enriquecen los aportes que brindan los diversos grupos humanos que comprenden nuestro hábitat y momento histórico.

Hoy en día podemos observar, como señala Castañeda (1999), cada vez mayor aceptación de la pareja homosexual en la sociedad heterosexual. La diversidad en la orientación sexual es un fenómeno presente de manera importante en nuestro país: de las casi 26 millones de familias que hay en México (25 millones 693 mil $584)$, casi $1 \%$ están conformadas por parejas del mismo sexo (229 mil 473), de acuerdo con cifras del Censo de Población y Vivienda (Consejo Nacional de Población, CONAPO, 2010), 
de las cuales más de 172 mil son de parejas homosexuales con hijos.

Por ejemplo, particularmente en nuestro estado, si bien no existen cifras exactas, se calcula que alrededor del $10 \%$ de la población es homosexual, la mitad de tal grupo vive en la capital, Mérida, y el resto en todo el interior del estado de Yucatán. En su mayoría son personas entre 15 y 50 años, aproximadamente (Izquierdo, 2009), por lo cual no se puede ignorar la existencia de las parejas con otras orientaciones sexuales en nuestro contexto.

Hicks y Platt (1970) consideran que la felicidad se define como un fenómeno personal y subjetivo, difícil de medir con herramientas válidas actualmente para los científicos sociales, por tal razón indican que la felicidad, el éxito, el ajuste y la satisfacción son los términos más utilizados con frecuencia en relación con el estado subjetivo de la relación marital.

Rivera Aragón (1992, citado por Concha, 2006), menciona que la satisfacción en la relación de pareja es una realidad que se construye por los miembros que la integran. Por su parte, López-lbor (1983, citado por Concha 2006), afirma que la satisfacción en la relación de pareja es un fenómeno dinámico, es decir, una pareja puede sentirse satisfecha en el presente, pero en el futuro puede deshacerse esa armonía percibida, y la que actualmente es considerada una relación rota, es posible que más adelante adquiera su equilibrio. Es necesario hacer hincapié en la importancia de las diferentes áreas que componen una relación de pareja. Es probable que en el círculo de la satisfacción, una pareja se encuentre satisfecha con una parte de su relación y menos satisfecha con otra.

Además, es importante señalar que existen diferentes tipos de pareja, por ejemplo, matrimonios o relaciones de noviazgo, entre otras. Dadas las características propias de cada una de ellas, es posible que los factores que contribuyan a la satisfacción en un tipo de relación no sean los mismos que en otro. Cabe mencionar que a pesar de haber encontrado información referente a la satisfacción, ninguna abarcaba a profundidad la satisfacción en el noviazgo. La mayoría de la literatura habla sobre satisfacción marital o bien de las parejas en general, sin especificar su estado. Si bien gran parte de los estudios de pareja se han dedicado a las parejas estables, generalmente casadas, el presente trabajo se centra en el noviazgo.

Como un antecedente de estudios sobre noviazgo y satisfacción en México y particularmente en el estado de Yucatán, se encuentra el realizado por Bargas Durán y Cortés Ayala (2009) en el estado de Yucatán, quienes llevaron a cabo un estudio exploratorio del significado psicológico de satisfacción en el noviazgo en estudiantes universitarios a través de la técnica de Redes Semánticas Naturales Modificadas, propuesta por la Dra. Reyes-Lagunes, y encontraron que tanto para hombres como para mujeres la satisfacción en el noviazgo se relaciona con el amor, así como también con la confianza, el respeto, la comunicación, la fidelidad y la comprensión. Sin embargo, las mujeres mencionan que también implica una relación de compromiso y de responsabilidad. Por otro lado, en los estudios realizados por Bargas y García en 2011, los resultados arrojan que los hombres están más satisfechos que las mujeres en Aislamiento vs. Intimidad y las mujeres están más satisfechas que los hombres en Comprensión-Apoyo, Interacción Positiva, Toma de Decisiones y Acuerdos, Interacción: Yo-Amigos de mi novio, Integración: Yo-Familia de mi novio, y por último Comunicación.

Sin embargo, estudios existentes en México que registren datos acerca de las relaciones con diversas orientaciones sexuales o la satisfacción en sus relaciones de noviazgo son prácticamente nulos, algunas de las tesis que abarcan algunos temas sobre relación de pareja son "Percepción de violencia, satisfacción con la relación y premisas sociocultural en parejas de novios" de Bagundo en 2006 y "Violencia en las 
relaciones de pareja, sexismo y enfrentamiento. Una perspectiva Psicosociocultural" de Cervantes y Vásquez en 2012.

Por lo tanto, el propósito de la investigación es conocer las diferencias de la satisfacción en las relaciones de noviazgo entre parejas heterosexuales y homosexuales en el municipio de Mérida en el estado de Yucatán. La pregunta general de investigación es: ¿Cuál es la diferencia en la satisfacción en el noviazgo según la orientación sexual (heterosexual/homosexual) y el género (femenino-mujer/masculino-hombre) en jóvenes de entre 18 y 30 años en la ciudad de Mérida, Yucatán? Esto es, determinar las diferencias en la satisfacción en el noviazgo entre los hombres y las mujeres, sin importar su orientación sexual, pero también encontrar cuáles son las dimensiones de la satisfacción en el noviazgo que son influidas por la orientación sexual.

\section{Método}

El tipo de investigación fue descriptivo por su alcance, ya que se pretendió descubrir, conocer y analizar las diferencias entre las dimensiones de la satisfacción del noviazgo en cada grupo de la muestra (orientación sexual, género). Por el tipo de escenario donde ocurrió es una investigación de campo y se realizó con jóvenes adultos que residen en la ciudad de Mérida, Yucatán. Por su temporalidad es de tipo trasversal debido a que se recolectaron datos en un momento concreto y, finalmente, por sus objetivos el estudio es de tipo piloto. En cuanto a su forma, el diseño de la investigación es de tipo no experimental, transeccional descriptivo, dado que la investigación se realizó sin manipular las variables y a que tuvo como objetivo indagar sobre las incidencias de las variables: género, orientación sexual y satisfacción, en la población objetivo.

\section{Participantes}

El muestreo fue no probabilístico por conveniencia. La muestra estuvo constituida por 90 participantes, con un rango de edad de entre 18 a 30 años $(M=22$ años y D.S.=3.104). En cuanto a su género, $48.88 \%$ (44) fueron hombres y el $51.11 \%$ (46) fueron mujeres. En cuanto a la orientación sexual $(52.22 \%, \mathrm{~N}=47)$ dijo ser heterosexual y $(47.77 \%, \mathrm{~N}=43)$ dijo tener una orientación sexual diferente.

\section{Variables}

Definición Conceptual:

Variable dependiente: Satisfacción.- Estado de placer de un organismo cuando ha logrado la meta de las tendencias motivadoras dominantes (Wolman, 2010).

Variable independiente 1: Género.- La condición de ser masculino y femenino o neutro. En un contexto humano, la distinción entre género y sexo refleja el uso de estos términos: sexo usualmente se refiere a los aspectos biológicos de masculinidad y feminidad, mientras que el género implica aspectos psicológico, comportamental, social y cultural de ser femenino o masculino (APA, 2006).

Variable independiente 2: Orientación sexual.- Es la atracción emocional, romántica, sexual o afectiva duradera hacia otros (APA, 2010).

\section{Definición operacional:}

Variable dependiente: Satisfacción.- Se define por la puntuación obtenida en los diferentes indicadores pertenecientes a la Escala Multidimensional de Satisfacción en el noviazgo (Bargas \& García, 2011).

Variable independiente 1: Género.- Se mide operacionalmente como la opción elegida, hombre o mujer, en la sección correspondiente de los datos generales. 
Variable independiente 2: Orientación sexual.Respuesta obtenida en la pregunta, ¿cómo te consideras? Que se encuentra en el apartado "Información general" en el instrumento utilizado y que tiene las opciones: heterosexual, preferentemente heterosexual, bisexual, preferentemente homosexual y homosexual.

\section{Hipótesis de trabajo}

- Las dimensiones de la satisfacción van a variar según la orientación sexual.

- Las dimensiones de la satisfacción en el noviazgo varían según el sexo.

La recolección de datos se realizó por medio del instrumento Ilamado Escala Multidimensional de Satisfacción en el Noviazgo, de Bargas y García (2011), el cual consiste en una escala tipo Likert pictográfica para medir la satisfacción en el noviazgo, compuesta por 11 dimensiones. En un primer grupo de ellas se miden aspectos relacionados con la relación de pareja al interior de la misma:

- Afinidad.- Percepción de similitud entre los miembros de la pareja reflejado en gustos, creencias, opiniones, intereses y actividades similares.

- Interacción positiva.- Actividades o acciones que son compartidas en la vida cotidiana de la pareja.

- Pasión.- Expresiones físicas de afecto dentro de la relación de pareja.

- Comprensión-Apoyo.- Percepción del apoyo que puede o no brindar la pareja en alguna situación compleja o difícil y el conocimiento que tiene la pareja de uno como persona.

- Comunicación.- Expresión verbal de intereses, actividades, problemas, etc., que se tiene entre los miembros de la pareja y la confianza existente entre ellos.
- Aislamiento vs. Intimidad.- Grado de retraimiento o lejanía que se puede tener con la pareja y la comodidad o incomodidad que esto pudiera representar.

- Toma de decisiones y acuerdos.- Reglas implícitas o explícitas que existen dentro de la relación de noviazgo para tomar una decisión o llegar a un acuerdo entre los miembros de una pareja.

Las otras dimensiones se centran en aspectos externos a la pareja como:

- Integración: Mi Novio(a)-Mi Familia.- Relación de la pareja de uno con la familia de uno mismo y, sobre todo, el grado de involucramiento o la integración que existe entre ellos.

- Integración:Yo-Familia de mi novio (a).- Relación que tiene uno mismo con la familia del novio(a), es decir, con la familia política.

- Interacción Yo-Amigos de mi novio (a).- Relación que tiene uno mismo como miembro de una pareja con las amistades directas del otro miembro.

- Interacción: Mi novio(a)-Mis amigos.- Relación que tiene el otro miembro de la pareja con los amigos de uno mismo.

\section{Instrumentos}

El instrumento está compuesto por 90 ítems y las respuestas de cada reactivo van en un continuo del 1 al 5, representadas por círculos de menor tamaño (nunca) a mayor tamaño (siempre). La confiabilidad del instrumento es el $57.88 \%$ de la varianza total acumulada. A continuación se presentan las 11 dimensiones de la satisfacción con sus respectivos coeficientes (de consistencia interna) alfa de Cronbach: 
Tabla 1.

Consistencias internas obtenidas para cada uno de los factores de la Escala Multidimensional de Satisfacción en el Noviazgo

\begin{tabular}{ll}
\hline Factores & Alfa \\
\hline Afinidad & 0.89 \\
Comprensión-Apoyo & 0.84 \\
Integración: Mi novio-mi familia & 0.84 \\
Interacción positiva & 0.76 \\
Aislamiento vs. Intimidad & 0.71 \\
Toma de decisiones y acuerdos & 0.74 \\
Pasión & 0.78 \\
Interacción: Yo-amigos de mi novio & 0.78 \\
Interacción: Mi novio-mis amigos & 0.74 \\
Integración: Yo-familia política & 0.79 \\
Comunicación & 0.75 \\
\hline
\end{tabular}

\section{Procedimiento}

La aplicación de instrumentos se realizó en lugares públicos de la ciudad de Mérida, como el zócalo de la ciudad, cafés, eventos que promueven la diversidad sexual. Para completar la muestra se aplicaron instrumentos en las instalaciones de la Facultad de Psicología (UADY) y por vía electrónica (internet) a personas conocidas que accedieron a responder de este modo. Previo a la entrega del instrumento, los participantes firmaron una carta de consentimiento informado en la cual autorizaban su participación voluntaria y donde se les garantizaba el uso confidencial de los resultados obtenidos de sus instrumentos. El criterio de inclusión de la muestra fue que los participantes se encontraran en una relación de noviazgo, sin importar el tiempo de su relación.

\section{Resultados}

Los resultados obtenidos, como se puede ver en la tabla 2, permiten constatar la existen- cia de diferencias significativas en la satisfacción en el noviazgo entre hombres y mujeres y entre personas con orientación sexual homosexual o heterosexual.

La primera hipótesis a analizar propone que la satisfacción en las dimensiones es diferente según la orientación sexual como se aprecia en la tabla 3. Para la comprobación de esta hipótesis, el estadístico utilizado fue la prueba Kruskal-Wallis. A continuación se explican las dimensiones que salieron significativas a un nivel $\alpha=0.05$.

Las dimensiones donde se presentaron las diferencias más importantes en la satisfacción según la orientación sexual fueron Integración: Mi novio-Mi familia, en la que se obtuvo una $\left(\chi^{2}=9.914,3\right)$ con una $p=0.019$, así como en la de Integración: Yo-Familia Política: $\left(\chi^{2}=9.041\right.$, $3)$, con una $p=0.029$. Lo anterior sugiere que los heterosexuales serían los más satisfechos en esta dimensión que los de otra orientación sexual, resaltando que los homosexuales no tuvieron una suma de rangos promedio baja. 


\section{Tabla 2.}

Consistencia interna, medias y desviaciones estándares obtenidas para cada uno de los factores de la Escala Multidimensional de Satisfacción en el Noviazgo (Bargas y García, 2011)

\begin{tabular}{llll}
\hline Dimensión & Media & D. S. & Alfa \\
\hline Afinidad & 3.3933 & 0.47376 & 0.457 \\
Comprensión y apoyo & 4.6494 & 0.42430 & 0.809 \\
Integración: mi novio(a) -mi familia & 4.1815 & 0.97107 & 0.880 \\
Interacción positiva & 4.2267 & 0.97107 & 0.188 \\
Aislamiento vs. Intimidad & 1.2800 & 0.47669 & 0.557 \\
Toma de decisiones y acuerdos & 4.4467 & 0.66166 & 0.661 \\
Pasión & 4.8926 & 0.37336 & 0.774 \\
Interacción: yo-amigos de mi novio (a) & 4.2044 & 0.80837 & 0.796 \\
Interacción: mi novio(a)-mis amigos & 3.8417 & 0.80837 & 0.546 \\
Integración: Yo-familia política & 4.2750 & 0.76387 & 0.686 \\
Comunicación & 4.7711 & 0.35164 & 0.570 \\
\hline
\end{tabular}

\section{Tabla 3.}

Diferencias obtenidas en los rangos entre las dimensiones de la satisfacción en el noviazgo y la orientación sexual según la prueba Kruskal-Wallis

\begin{tabular}{lcccc}
\hline & & \multicolumn{2}{c}{ Orientación sexual } \\
\hline Dimensiones de la satisfacción & Heterosexual & Bisexual & $\begin{array}{c}\text { Preferentemente } \\
\text { homosexual }\end{array}$ & Homosexual \\
Afinidad & 44.79 & 38.19 & 56.83 & 47.31 \\
Comprensión y apoyo & 50.02 & 34.63 & 39.33 & 42.16 \\
Integración: mi novio-mi familia & $52.71^{*}$ & $25.94^{*}$ & $37.83^{*}$ & $40.52^{*}$ \\
Interacción positiva & 44.10 & 39.06 & 29.83 & 50.64 \\
Aislamiento vs. Intimidad & 40.90 & 61.88 & 62.17 & 46.59 \\
Toma de decisiones y acuerdos & 47.27 & 39.06 & 52.33 & 43.88 \\
Pasión & 47.21 & 52 & 59.50 & 42.02 \\
Interacción: Yo -amigos de mi novio & 45.65 & 43.13 & 59.67 & 40.67 \\
Interacción: Mi novio-mis amigos & 41.34 & 46.06 & $14.17^{*}$ & 45.55 \\
Integración: Yo-familia política & $51.56^{*}$ & $35.63^{*}$ & 50.33 & $42^{*}$ \\
Comunicación & 47.35 & 43.88 & 42.73 \\
\hline
\end{tabular}

${ }^{*} p \leq 0.05{ }^{* *} p \leq 0.001$ 
La segunda hipótesis plantea que la satisfacción en las dimensiones cambia según el sexo. El estadístico para comprobar esta hipótesis es la $t$ de Student para muestras independientes, pero dado que la muestra no cumplía con los criterios de normalidad requeridos para el uso de esta prueba, se decidió utilizar el estadístico de prueba $U$ de Mann-Whitney para analizar los datos.
En cuanto a las dimensiones que resultaron estadísticamente significativas a un nivel $\alpha=$ 0.05 , según el sexo, se presentaron en las dimensiones de Interacción positiva: en la que se obtuvo una $Z=-2.806$ con una $p=0.005$; Toma de decisiones y acuerdos con una $Z=-2.973$ a $p=0.003$; Interacción: Yo-Amigos de mi novio(a) en la que se encontró una $Z=-1.999$ con una $p=0.046$ y en la de Comunicación donde se obtuvo una $Z=-2.738$ con una $p=0.006$.

Tabla 4.

Diferencias obtenidas en los rangos entre las dimensiones de la satisfacción en el noviazgo y el sexo por la prueba $U$ de Mann-Whitney, sin tomar en cuenta la orientación sexual.

\begin{tabular}{lll}
\hline & & Sexo \\
\hline Dimensiones & Hombre & 42.39 \\
Afinidad & 48.75 & 49.01 \\
Comprensión y apoyo & 41.83 & 45.95 \\
Integración: mi novio-mi familia & 45.03 & $39.04^{*}$ \\
Interacción positiva & $52.25^{*}$ & 41.16 \\
Aislamiento vs. Intimidad & 50.03 & $53.24^{*}$ \\
Toma de decisiones y acuerdos & $37.42^{*}$ & 46.13 \\
Pasión & 44.84 & $50.83^{*}$ \\
Interacción: yo-amigos de mi novio & $39.93^{*}$ & 46.36 \\
Interacción: Mi novio-mis amigos & 44.60 & 49.35 \\
Integración: Yo-familia política & 41.48 & $52.11^{*}$ \\
Comunicación & $38.59^{*}$ &
\end{tabular}

En cuanto a las diferencias significativas en la dimensión Interacción positiva, se infiere que los hombres están más satisfechos en el tiempo que comparten con su pareja en comparación con las mujeres. Sobre la dimensión Toma de decisiones y acuerdos, las mujeres están más satisfechas con las reglas y acuerdos dentro de su relación que los hombres. Por otro lado, las mujeres nuevamente, se encuentran más satisfechas con respecto a la interacción
Yo-Amigos de mi novio(a), es decir, están satisfechas con la relación que tienen con los amigos de su pareja, así como en la dimensión de Comunicación donde ellas se encuentran más satisfechas en lo relativo a la calidad de comunicación que tienen en su relación, sintiéndose más cómodas cuando hablan con su pareja de sus problemas, intereses y metas. 


\section{Discusión}

Este estudio mostró que el grado de satisfacción de acuerdo con la orientación sexual varía según las dimensiones. En este sentido, las dimensiones significativas fueron Integración: Yo-Familia de mi novio(a) y la dimensión Integración: Mi Novio-Mi Familia. Una posible explicación sería, que en el caso de las personas con orientación homosexual aún existe cierto rechazo por parte de sus familias hacia la relación que existe entre ellos. En la literatura Powers y Ellis (1999), mencionan que las personas de distinta orientación sexual pueden optar por hacerle frente a su familia sacando el tema a colación de manera eficaz aunque lamentablemente, también es muy común que los integrantes de la pareja se distancien de la familia.

En este sentido, para Castañeda (1999), una de las dificultades que enfrenta la pareja homosexual es el aislamiento. La mayoría de los homosexuales vive más o menos distanciados de su familia de origen. La pareja se tiene que sostener por sí sola, sin los vínculos afectivos y sociales, las actividades y los proyectos que forman y sustentan la vida familiar. Muchas familias tratarán de enterrar el asunto conduciéndose como si el hijo no hubiera dicho nada, nunca mencionarán el tema ni le harán preguntas al respecto ni invitarán a la pareja de sus hijos(as) a las reuniones familiares, ni la tomarán en cuenta de manera alguna. Este tipo de reacción es profundamente hiriente para quien presenta una orientación sexual distinta porque invalida, como si no existiera, una parte central de su vida: su relación de pareja (Castañeda, 1999).

Según los resultados encontrados en esta investigación, este distanciamiento de sus familias se puede deber a que no encuentran en ellas la misma satisfacción que encuentran con su pareja, por lo cual las personas con diferente orientación sexual deciden alejarse de sus familias y pasar más tiempo con su pareja, ya que ésta es importante para ellos y pueden encontrar en ella vínculos afectivos y aceptación que no les es fácil encontrar en sus familias. Asimismo, debido a que la familia tiende a rechazar a la pareja al no tomarla en cuenta, esto puede causar que las parejas se alejen de sus familias de origen y no encuentren apoyo en ellas, lo cual puede explicar la razón de las puntuaciones bajas encontradas en esta investigación respecto a las dimensiones relacionadas con la familia. Un factor que pudo haber influido en los resultados es el tiempo de relación, ya que las relaciones homosexuales fueron menos duraderas que las heterosexuales. Es probable que dicha variable esté relacionada con las diferencias en las interacciones familiares con distinta orientación sexual, debido a que no se logra establecer una buena relación familiar por el corto tiempo de relación.

Por otro lado, independientemente de la orientación sexual, en la dimensión Interacción positiva, se observa que probablemente los hombres estén más satisfechos en situaciones como tomarse de la manos, disfrutar el tiempo que pasan juntos y se sienten bien, disfrutan platicar, besar a su pareja y también les gustan las actividades que realizan juntos. En la dimensión Interacción: yo-amigos de mi novio(a), se observa que las mujeres podrían estar más satisfechas en la relación que tienen con los amigos de su pareja probablemente porque las mujeres tienden a acercarse más a las amistades de su pareja en comparación con los hombres, quienes no suelen interactuar mucho con los amigos de sus parejas.

En relación con la tercera dimensión de Comunicación, los resultados señalan que las mujeres están más satisfechas en su relación, ya que disfrutan el tiempo en que platican los temas que son de su interés, lo que quisieran hacer en la vida, lo que hicieron en el día y/o los problemas que tienen. Según Allen y Thomson, (1984, citado en Quintal, 1995), los teóricos interesados en el estudio de esta variable coinciden en que una buena comunicación entre los miembros de una pareja contribuye al incremento de la satisfacción marital y es requisito 
indispensable para la resolución de conflictos. Se hace la observación que, independientemente de quién esté más satisfecho con la comunicación, el hecho de que ésta exista en una relación es vital para el mantenimiento de ella, tal como dice Kawamoto, (1985, citado en Díaz-Loving, 1999): la comunicación asertiva se introdujo como un método de comunicación para el mantenimiento de las relaciones interpersonales, entre ellas las de pareja. Flores y Díaz-Loving (2002), señalan la importancia del papel de la comunicación asertiva en la pareja, puesto que es importantísimo homogenizar los códigos de comunicación, ya que con ello la pareja podrá sustituir las conductas pasivas y agresivas que producen incomunicación, por conductas y actitudes asertivas que facilitan la mutua compresión, el respeto y la toma responsable de decisiones. Sin embargo, Díaz-Loving (1999), señala que conseguir en la relación de pareja que la comunicación y el diálogo con el entorno se realicen de manera natural, espontánea, fluida, sin malos entendidos y sin conflictos, es más difícil de lo que a primera vista pudiera parecer.

Se observa que en la cuarta y última dimensión denominada Toma de decisiones y acuerdos, son de nuevo las mujeres las que estuvieron probablemente más satisfechas. Se sugiere que esto se debe a que ellas hacen uso de la comunicación asertiva, es decir, expresan de manera adecuada sus inquietudes, sus expectativas y planes; comparten de manera más clara las cosas de su interés, sus metas en la vida, los problemas personales que sólo le contarían a su pareja.

Finalmente, en cuanto al desarrollo del estudio es importante señalar que éste no estuvo exento de dificultades. Por ejemplo, la muestra requerida para representar a las mujeres con orientación homosexual fue difícil de encontrar debido a que ellas son reservadas con respecto a su orientación sexual y situación sentimental. Cabe mencionar que al no haber asociaciones lésbicas resultó muy difícil encontrar a mujeres que pudieran apoyar a confirmar esta muestra, a diferencia de la homosexualidad varonil que congrega diversas asociaciones civiles dirigidas por hombres para hombres; la participación de las mujeres era nula.

Dentro de los principales resultados que brinda esta investigación se puede inferir que aún se tiene, de cierta forma, un rechazo hacia las parejas de personas con distinta orientación sexual a la heterosexual, la cual se ve reflejada en las familias de éstos. Otro resultado consiste en que esta investigación permite conocer nuevos datos acerca de las satisfacción en el género masculino; ya que en los resultados de la tesis de Bargas y García (2011), las mujeres demostraron mayor satisfacción en la interacción positiva y por lo contario en este estudio los hombres fueron los más satisfechos en esta dimensión, se considera que tal situación se debe a la adición de una nueva variable, la cual fue la orientación sexual. También esta investigación permite afirmar o sustentar resultados anteriores como la dimensión Toma de decisiones y acuerdos, la cual en los dos estudios se encontró significativa.

Por último, se sugiere que para estudios futuros sería interesante anexar nuevas variables por ejemplo: tiempo de relación, religión, nivel socioeconómico, etc. Estas variables podrían arrojar nuevas perspectivas a los resultados, al enfatizar la diversidad en la orientación sexual. Por último, se sugiere realizar esta investigación con una muestra más grande que permita profundizar en los resultados.

Como conclusión podría decirse que la presente investigación permite entender más a fondo la dinámica actual del noviazgo en los jóvenes y comprender mejor las relaciones de pareja que se dan en la actualidad. La satisfacción podría estar relacionada con la situación actual de violencia en el noviazgo en Yucatán. Los estudios sobre la relación de pareja y de noviazgo muestran una situación alarmante por el alto número de casos que la presentan: en un estudio realizado en el 2012 por la En- 
cuesta Nacional de la Juventud, un $4.4 \%$ de los jóvenes yucatecos de entre 12 y 29 años que vive una relación de noviazgo ha reconocido la existencia de golpes o agresiones físicas en su relación. Otro $7.1 \%$ refirió que está sujeto a insultos o amenazas y de ese grupo, por lo menos $20.7 \%$ comentó que los insultos y amenazas son frecuentes. Es por eso que es importante conocer la situación actual en los noviazgos de los adultos jóvenes de la ciudad de Mérida; según los datos obtenidos podría elaborarse un programa para la prevención de la situación de violencia en estas parejas, con el objetivo de lograr relaciones sanas, satisfactorias, duraderas, constructivas, funcionales y amorosas, partiendo del supuesto de que la satisfacción en la pareja, independientemente de la orientación sexual, es indispensable para establecer relaciones sanas.
American Psychological Association. (2010). Publication manual of the American Psychological Association (6th ed.). Washingto, D.C.: APA.

American Psychological Association. (2006). APA dictionary of Psychology. Washingto,D.C.: APA

Ardila, R. (2008). Problemas y Perspectivas: En Homosexualidad y Psicología. Bogotá: Manual Moderno.

Bagundo, I. (2006). Percepción de violencia, satisfacción con la relación y premisas socioculturales en parejas de novios. Tesis de Licenciatura no publicada. Facultad de Psicología, Universidad Autónoma de Yucatán. México.

Bargas,E. \& García, A. (2011). Satisfacción en el noviazgo: construcción y validación de un instrumento. Tesis de Licenciatura no publicada. Facultad de Psicología, Universidad Autónoma de Yucatán, México.

Bargas Durán, E. \& Cortés Ayala, L. (2009). Amor y Noviazgo: Significado psicológico en jóvenes. Ponencia en el III Congreso Nacional de Investigación en Salud y Sociedad, Mérida, Yucatán; Centro de Investigaciones Regionales de la Universidad Autónoma de Yucatán, Mérida, México.

Castañeda, M. (1999). La experiencia homosexual: Para comprender la homosexualidad desde dentro y desde fuera. Toluca, México: Paidós.

Cervantes, S. \& Vásquez, M. (2012). Violencia en las relaciones de pareja, sexismo y enfrentamiento. Una perspectiva psicosociocultural. Tesis de Licenciatura no publicada. Facultad de Psicología. Universidad Autónoma de Yucatán. México.

Concha Canul, E. F. (2006). Premisas socioculturales, estilos de apego, celos y su relación con la satisfacción de lo pareja en el noviazgo. Tesis de licenciatura no publicada. Universidad Autónoma de Yucatán, facultad de Psicología. Mérida, Yucatán.

Consejo Nacional de Población, CONAPO. (2010). La situación demográfica en México 2010. México: CONAPO.

Diaz-Loving R. (1999). Antología psicosocial de la pareja. México: Asociación mexicana de psicología social.

Flores, M. M. \& Díaz-Loving, R. (2002). Asertividad: Una alternativa para el óptimo manejo de las relaciones interpersonales. México: Universidad Autónoma de Yucatán.

Hicks, M. \& Platt. R. (1970). Marital happiness and stability. A review of the research in the sixties. Journal of Marriage and the Family, 32 (4), 533- 574.

Izquierdo, L. (2009, julio 10). Doble vida, resultado de la presión social y la discriminación a homosexuales y lesbianas en Yucatán [Blog en línea]. Recuperado de http://a7.com.mx/reportajes/1117-lapresion-social-y-doble-vida-resultado-de-la-presión-social-y-ladiscrimianion-a-homosexuales-y-lesbianas-en-yucatan.html

López-Parra, S., Rivera-Aragón, S., García-Méndez, M. \& Vargas-Núñez, I. (2010). ¿Qué genera conflicto en el noviazgo?: Diferencias por sexo y escolaridad. La Psicología Social en México, XIII, 245-250. México: AMEPSO.

Nicolau, S., Martell, J., \& Méndez J. (2004). Módulo para la prevención de la violencia en las relaciones de pareja de l@s jóvenes; Bogotá: ALVI Impresores.

Pichardo, J. (2009). Entender la diversidad familiar. Barcelona: Edicions bellaterra.

Powers, B. \& Ellis, A. (1999). Acéptate, acéptalo: cómo explicar y comprender las distintas orientaciones sexuales. Buenos Aires: Paidós.

Quintal Acereto, S. (1995). Satisfación marital: investigaciones y factores que la determinan. Tesis de licenciatura no publicada. Facultad de Psicología, Universidad Autónoma de Yucatán.

Villagómez, G. (2010). Violencia en el noviazgo y matrimonio. Mérida, Yucatán, México, Universidad Autónoma de Yucatán.

Wolman B., (2010).Diccionario de psicología. México: Trillas 